\title{
A detailed study on a new
} the Caputo-Fabrizio time-fractional derivative

\author{
K. Hosseini ${ }^{1 *}$, M. Ilie ${ }^{1}$, M. Mirzazadeh² and D. Baleanu ${ }^{3,4}$
}

\section{"Correspondence:}

kamyar_hosseini@yahoo.com

${ }^{1}$ Department of Mathematics, Rasht

Branch, Islamic Azad University,

Rasht, Iran

Full list of author information is

available at the end of the article

\begin{abstract}
The present article aims to present a comprehensive study on a nonlinear time-fractional model involving the Caputo-Fabrizio (CF) derivative. More explicitly, a new $(2+1)$-dimensional mKdV (2D-mKdV) equation involving the Caputo-Fabrizio time-fractional derivative is considered and an analytic approximation for it is retrieved through a systematic technique, called the homotopy analysis transform (HAT) method. Furthermore, after proving the Lipschitz condition for the kernel $\psi(x, y, t ; u)$, the fixed-point theorem is formally utilized to demonstrate the existence and uniqueness of the solution of the new 2D-mKdV equation involving the CF time-fractional derivative. A detailed study finally is carried out to examine the effect of the Caputo-Fabrizio operator on the dynamics of the obtained analytic approximation.
\end{abstract}

Keywords: $(2+1)$-dimensional mKdV equation; Caputo-Fabrizio time-fractional derivative; Homotopy analysis transform method; Analytic approximation; Fixed-point theorem; Existence and uniqueness of the solution 
There are different variations of the classical KdV equation, some of those reported in the literature are $[2,3]$ :

$$
\begin{array}{ll}
u_{t}+\alpha_{1} u_{x x x}+\alpha_{2} u^{2} u_{x}=0, & (\text { modified } \mathrm{KdV}), \\
u_{t}+\alpha_{1} u_{x x x}+\alpha_{2} u^{n} u_{x}=0, & (\text { generalized KdV) }
\end{array}
$$

and the $(2+1)$-dimensional $\mathrm{KdV}$ equation

$$
\left\{\begin{array}{l}
u_{t}-u_{x x x}+3(u v)_{x}=0, \\
u_{x}-v_{y}=0
\end{array}\right.
$$

Many efforts have been devoted to studying the KdV-type equations, for example, Wazwaz [2] used different reliable methods to obtain solitons and periodic solutions of the KdV, modified $\mathrm{KdV}$, and generalized $\mathrm{KdV}$ equations, and Wang [3] derived lump solutions of the $(2+1)$-dimensional $\mathrm{KdV}$ equation by means of an ansatz based on the quadratic functions. Recently, the fractional forms of the KdV-type equations have been explored using a series of systematic methods in $[4,5]$.

Our aim of this paper is studying a new $(2+1)$-dimensional $\mathrm{mKdV}$ equation involving the Caputo-Fabrizio time-fractional derivative as follows:

$$
{ }_{0}^{\mathrm{CF}} D_{t}^{\alpha} u=6 u^{2} u_{x}-6 u^{2} u_{y}+u_{x x x}-u_{y y y}-3 u_{x x y}+3 u_{x y y}, \quad 0<\alpha \leq 1
$$

through a systematic technique called the homotopy analysis transform method [6-11]. The classical form of the new 2D-mKdV Eq. (1) was first proposed by Wang and Kara [12] in 2019 using the extended Lax pair. Wang and Kara in [12] extracted a group of solitary wave solutions of the new 2D-mKdV equation (its classical form) through the Lie symmetry method.

Recently, nonlinear ODEs/PDEs involving the Caputo-Fabrizio fractional derivative have gained significant attention owing to their potential to describe many complicated physical phenomena. In this respect, Shah et al. [13] analyzed a nonlinear model of dengue fever disease with the CF fractional derivative using the Laplace Adomian decomposition method. Owolabi and Atangana [14] explored a series of nonlinear fractional parabolic differential equations involving the CF derivative thought a numerical scheme. In another work performed by Arshad et al. [15], the CD4+ T-cells model of HIV infection with the CF fractional derivative was studied using an effective numerical scheme. Shaikh et al. [16] employed the iterative Laplace transform method to analyze a group of fractional reaction-diffusion equations involving the $\mathrm{CF}$ derivative. More articles can be found in [17-45].

The rest of this paper is as follows: In Sect. 2, the Caputo-Fabrizio fractional operators are reviewed in detail. In Sect. 3, the Lipschitz condition for the kernel $\psi(x, y, t ; u)$ is proved, then the fixed-point theorem is formally applied to show the existence and uniqueness of the solution of the new $2 \mathrm{D}-\mathrm{mKdV}$ equation involving the $\mathrm{CF}$ time-fractional derivative. In Sect. 4, an analytic approximation for the new 2D-mKdV equation involving the Caputo-Fabrizio time-fractional derivative is acquired using the HAT method. The results of this paper are summarized in the last section. 


\section{Basic definitions and features}

This section presents the basic definitions and features of the Caputo-Fabrizio fractional operators. In this respect, first the Caputo-Fabrizio fractional derivative and integral are defined, then the Laplace transform of the Caputo-Fabrizio fractional derivative is given.

Definition 1 Suppose that $u(t) \in H^{1}(a, b), b>a$ and $\alpha \in(0,1]$. Then, the Caputo-Fabrizio fractional derivative of $u(t)$ of order $\alpha$ is given by [17]

$$
{ }_{a}^{\mathrm{CF}} D_{t}^{\alpha} u(t)=\frac{M(\alpha)}{1-\alpha} \int_{a}^{t} u^{\prime}(\varepsilon) e^{-\frac{\alpha}{1-\alpha}(t-\varepsilon)} d \varepsilon
$$

where $M(\alpha)$ is a normalized function satisfying $M(0)=M(1)=1$.

Definition 2 The Caputo-Fabrizio fractional integral of $u(t)$ of order $\alpha(\alpha \in(0,1])$ is given by [18]

$$
{ }_{0}^{\mathrm{CF}} I_{t}^{\alpha} u(t)=\frac{2(1-\alpha)}{(2-\alpha) M(\alpha)} u(t)+\frac{2 \alpha}{(2-\alpha) M(\alpha)} \int_{0}^{t} u(\varepsilon) d \varepsilon, \quad t \geq 0 .
$$

Definition 3 The Laplace transform of ${ }_{0}^{\mathrm{CF}} D_{t}^{\alpha} u(t)$ is given as [17]

$$
\mathcal{L}\left[{ }_{0}^{\mathrm{CF}} D_{t}^{\alpha} u(t)\right](s)=\frac{s \mathcal{L}[u(t)](s)-u(0)}{s+\alpha(1-s)}
$$

and in the general case, we have

$$
\mathcal{L}\left[{ }_{0}^{\mathrm{CF}} D_{t}^{(\alpha+n)} u(t)\right](s)=\frac{s^{n+1} \mathcal{L}[u(t)](s)-s^{n} u(0)-s^{n-1} u^{\prime}(0)-\cdots-u^{(n)}(0)}{s+\alpha(1-s)}
$$

Theorem 1 The following Lipschitz condition holds for the Caputo-Fabrizio fractional derivative given in Definition 1:

$$
\left\|{ }_{a}^{\mathrm{CF}} D_{t}^{\alpha} u(t)-{ }_{a}^{\mathrm{CF}} D_{t}^{\alpha} v(t)\right\| \leq \lambda\|u(t)-v(t)\| .
$$

Proof In a similar manner as in [23], it is easy to show that

$$
\begin{aligned}
\left\|{ }_{a}^{\mathrm{CF}} D_{t}^{\alpha} u(t)-{ }_{a}^{\mathrm{CF}} D_{t}^{\alpha} \nu(t)\right\| & =\left\|\frac{M(\alpha)}{1-\alpha} \int_{a}^{t} u^{\prime}(\varepsilon) e^{-\frac{\alpha}{1-\alpha}(t-\varepsilon)} d \varepsilon-\frac{M(\alpha)}{1-\alpha} \int_{a}^{t} v^{\prime}(\varepsilon) e^{-\frac{\alpha}{1-\alpha}(t-\varepsilon)} d \varepsilon\right\| \\
& =\left\|\frac{M(\alpha)}{1-\alpha}\left(\int_{a}^{t} u^{\prime}(\varepsilon) e^{-\frac{\alpha}{1-\alpha}(t-\varepsilon)} d \varepsilon-\int_{a}^{t} v^{\prime}(\varepsilon) e^{-\frac{\alpha}{1-\alpha}(t-\varepsilon)} d \varepsilon\right)\right\| \\
& =\left\|\frac{M(\alpha)}{1-\alpha} e^{-\frac{\alpha}{1-\alpha}(t-\varepsilon)}\left(\int_{a}^{t}\left(u^{\prime}(\varepsilon)-v^{\prime}(\varepsilon)\right) d \varepsilon\right)\right\| \\
& \leq \frac{M(\alpha)}{1-\alpha} \mu e^{-\frac{\alpha}{1-\alpha}(t-\alpha)}\|u(t)-v(t)\| \\
& =\lambda\|u(t)-v(t)\| .
\end{aligned}
$$




\section{The model and the existence and uniqueness of its solution}

To start, let us consider

$$
\psi(x, y, t ; u)=6 u^{2} u_{x}-6 u^{2} u_{y}+u_{x x x}-u_{y y y}-3 u_{x x y}+3 u_{x y y} .
$$

This suggests that Eq. (1) can be rewritten as

$$
{ }_{0}^{\mathrm{CF}} D_{t}^{\alpha} u(x, y, t)=\psi(x, y, t ; u)
$$

Applying the CF fractional integral to both sides of Eq. (2) results in

$$
u(x, y, t)-u(x, y, 0)=\frac{2(1-\alpha)}{(2-\alpha) M(\alpha)} \psi(x, y, t ; u)+\frac{2 \alpha}{(2-\alpha) M(\alpha)} \int_{0}^{t} \psi(x, y, \varepsilon ; u) d \varepsilon
$$

In order to show that the kernel $\psi(x, y, t ; u)$ satisfies the Lipschitz condition, we first consider bounded functions $u(x, y, t)$ and $v(x, y, t)$. Using the triangle property of norms, one can find

$$
\begin{aligned}
&\|\psi(x, y, t ; u)-\psi(x, y, t ; v)\| \\
&=\|\left(6 u^{2} u_{x}-6 v^{2} v_{x}\right)-\left(6 u^{2} u_{y}-6 v^{2} v_{y}\right)+\left(u_{x x x}-v_{x x x}\right)-\left(u_{y y y}-v_{y y y}\right) \\
&-\left(3 u_{x x y}-3 v_{x x y}\right)+\left(3 u_{x y y}-3 v_{x y y}\right) \| \\
&= \| 2 \frac{\partial}{\partial x}\left(u^{3}-v^{3}\right)-2 \frac{\partial}{\partial y}\left(u^{3}-v^{3}\right)+\frac{\partial^{3}}{\partial x^{3}}(u-v)-\frac{\partial^{3}}{\partial y^{3}}(u-v) \\
&-3 \frac{\partial^{3}}{\partial y \partial x^{2}}(u-v)+3 \frac{\partial^{3}}{\partial y^{2} \partial x}(u-v) \| \\
& \leq 2\left\|\frac{\partial}{\partial x}\left(u^{3}-v^{3}\right)\right\|+2\left\|\frac{\partial}{\partial y}\left(u^{3}-v^{3}\right)\right\|+\left\|\frac{\partial^{3}}{\partial x^{3}}(u-v)\right\|+\left\|\frac{\partial^{3}}{\partial y^{3}}(u-v)\right\| \\
&+3\left\|\frac{\partial^{3}}{\partial y \partial x^{2}}(u-v)\right\|+3\left\|\frac{\partial^{3}}{\partial y^{2} \partial x}(u-v)\right\| \\
& \leq 2 A\left\|u^{3}-v^{3}\right\|+2 B\left\|u^{3}-v^{3}\right\|+C\|u-v\|+D\|u-v\|+3 E\|u-v\|+3 F\|u-v\| \\
& \leq(2 A+2 B)\left(\mu^{2}+\mu v+v^{2}\right)\|u-v\|+C\|u-v\|+D\|u-v\|+3 E\|u-v\|+3 F\|u-v\| \\
&=\left((2 A+2 B)\left(\mu^{2}+\mu v+v^{2}\right)+C+D+3 E+3 F\right)\|u-v\|, \quad\|u\| \leq \mu,\|v\| \leq v .
\end{aligned}
$$

Therefore

$$
\|\psi(x, y, t ; u)-\psi(x, y, t ; v)\| \leq \lambda\|u-v\|,
$$

which

$$
\lambda=\left((2 A+2 B)\left(\mu^{2}+\mu v+v^{2}\right)+C+D+3 E+3 F\right) .
$$

This confirms that the Lipschitz condition is satisfied for the kernel $\psi(x, y, t ; u)$. 
Now, based on the Eq. (3) and the fixed-point theorem, an iterative scheme is established as follows:

$$
u_{n+1}(x, y, t)=\frac{2(1-\alpha)}{(2-\alpha) M(\alpha)} \psi\left(x, y, t ; u_{n}\right)+\frac{2 \alpha}{(2-\alpha) M(\alpha)} \int_{0}^{t} \psi\left(x, y, \varepsilon ; u_{n}\right) d \varepsilon
$$

where

$$
u_{0}(x, y, t)=u(x, y, 0)
$$

It is clear that

$$
\begin{aligned}
e_{n}(x, y, t)= & u_{n}(x, y, t)-u_{n-1}(x, y, t) \\
= & \frac{2(1-\alpha)}{(2-\alpha) M(\alpha)}\left(\psi\left(x, y, t ; u_{n-1}\right)-\psi\left(x, y, t ; u_{n-2}\right)\right) \\
& +\frac{2 \alpha}{(2-\alpha) M(\alpha)} \int_{0}^{t}\left(\psi\left(x, y, \varepsilon ; u_{n-1}\right)-\psi\left(x, y, \varepsilon ; u_{n-2}\right)\right) d \varepsilon
\end{aligned}
$$

and

$$
u_{n}(x, y, t)=\sum_{i=0}^{n} e_{i}(x, y, t) .
$$

Theorem 2 If the function $u(x, y, t)$ is bounded, then

$$
\left\|e_{n}(x, y, t)\right\| \leq\left(\frac{2(1-\alpha)}{(2-\alpha) M(\alpha)} \lambda+\frac{2 \alpha}{(2-\alpha) M(\alpha)} \lambda t\right)^{n}\|u(x, y, 0)\| .
$$

Proof by induction Suppose that $n=1$. Then, one can write

$$
\begin{aligned}
\left\|e_{1}(x, y, t)\right\|= & \left\|u_{1}(x, y, t)-u_{0}(x, y, t)\right\| \\
\leq & \frac{2(1-\alpha)}{(2-\alpha) M(\alpha)}\left\|\psi\left(x, y, t ; u_{0}\right)-\psi\left(x, y, t ; u_{-1}\right)\right\| \\
& +\frac{2 \alpha}{(2-\alpha) M(\alpha)} \int_{0}^{t}\left\|\psi\left(x, y, \varepsilon ; u_{0}\right)-\psi\left(x, y, \varepsilon ; u_{-1}\right)\right\| d \varepsilon \\
\leq & \frac{2(1-\alpha)}{(2-\alpha) M(\alpha)} \lambda\left\|u_{0}-u_{-1}\right\|+\frac{2 \alpha}{(2-\alpha) M(\alpha)} \int_{0}^{t} \lambda\left\|u_{0}-u_{-1}\right\| d \varepsilon \\
= & \frac{2(1-\alpha)}{(2-\alpha) M(\alpha)} \lambda\|u(x, y, 0)\|+\frac{2 \alpha}{(2-\alpha) M(\alpha)} \int_{0}^{t} \lambda\|u(x, y, 0)\| d \varepsilon \\
= & \frac{2(1-\alpha)}{(2-\alpha) M(\alpha)} \lambda\|u(x, y, 0)\|+\frac{2 \alpha}{(2-\alpha) M(\alpha)} \lambda\|u(x, y, 0)\| \int_{0}^{t} d \varepsilon \\
= & \frac{2(1-\alpha)}{(2-\alpha) M(\alpha)} \lambda\|u(x, y, 0)\|+\frac{2 \alpha}{(2-\alpha) M(\alpha)} \lambda\|u(x, y, 0)\| t \\
= & \left(\frac{2(1-\alpha)}{(2-\alpha) M(\alpha)} \lambda+\frac{2 \alpha}{(2-\alpha) M(\alpha)} \lambda t\right)\|u(x, y, 0)\| .
\end{aligned}
$$


Now, if the relation holds for $n-1$, namely

$$
\left\|e_{n-1}(x, y, t)\right\| \leq\left(\frac{2(1-\alpha)}{(2-\alpha) M(\alpha)} \lambda+\frac{2 \alpha}{(2-\alpha) M(\alpha)} \lambda t\right)^{n-1}\|u(x, y, 0)\|,
$$

then, we will prove that

$$
\left\|e_{n}(x, y, t)\right\| \leq\left(\frac{2(1-\alpha)}{(2-\alpha) M(\alpha)} \lambda+\frac{2 \alpha}{(2-\alpha) M(\alpha)} \lambda t\right)^{n}\|u(x, y, 0)\| .
$$

To show this, we proceed as follows:

$$
\begin{aligned}
& \left\|e_{n}(x, y, t)\right\|=\left\|u_{n}(x, y, t)-u_{n-1}(x, y, t)\right\| \\
& \leq \frac{2(1-\alpha)}{(2-\alpha) M(\alpha)}\left\|\psi\left(x, y, t ; u_{n-1}\right)-\psi\left(x, y, t ; u_{n-2}\right)\right\| \\
& +\frac{2 \alpha}{(2-\alpha) M(\alpha)} \int_{0}^{t}\left\|\psi\left(x, y, \varepsilon ; u_{n-1}\right)-\psi\left(x, y, \varepsilon ; u_{n-2}\right)\right\| d \varepsilon \\
& \leq \frac{2(1-\alpha)}{(2-\alpha) M(\alpha)} \lambda\left\|u_{n-1}-u_{n-2}\right\|+\frac{2 \alpha}{(2-\alpha) M(\alpha)} \int_{0}^{t} \lambda\left\|u_{n-1}-u_{n-2}\right\| d \varepsilon \\
& =\frac{2(1-\alpha)}{(2-\alpha) M(\alpha)} \lambda\left\|e_{n-1}(x, y, t)\right\|+\frac{2 \alpha}{(2-\alpha) M(\alpha)} \int_{0}^{t} \lambda\left\|e_{n-1}(x, y, \varepsilon)\right\| d \varepsilon \\
& =\left(\frac{2(1-\alpha)}{(2-\alpha) M(\alpha)} \lambda+\frac{2 \alpha}{(2-\alpha) M(\alpha)} \lambda t\right)^{n-1}\|u(x, y, 0)\| \\
& \times\left(\frac{2(1-\alpha)}{(2-\alpha) M(\alpha)} \lambda+\frac{2 \alpha}{(2-\alpha) M(\alpha)} \lambda \int_{0}^{t} d \varepsilon\right) \\
& =\left(\frac{2(1-\alpha)}{(2-\alpha) M(\alpha)} \lambda+\frac{2 \alpha}{(2-\alpha) M(\alpha)} \lambda t\right)^{n-1} \\
& \times\left(\frac{2(1-\alpha)}{(2-\alpha) M(\alpha)} \lambda+\frac{2 \alpha}{(2-\alpha) M(\alpha)} \lambda t\right)\|u(x, y, 0)\| \\
& =\left(\frac{2(1-\alpha)}{(2-\alpha) M(\alpha)} \lambda+\frac{2 \alpha}{(2-\alpha) M(\alpha)} \lambda t\right)^{n}\|u(x, y, 0)\| \text {. }
\end{aligned}
$$

Theorem 3 If at $t=t_{0}$ we have

$$
0 \leq \frac{2(1-\alpha)}{(2-\alpha) M(\alpha)} \lambda+\frac{2 \alpha}{(2-\alpha) M(\alpha)} \lambda t_{0}<1
$$

then the solution of the new $2 D-m K d V$ equation involving the CF time-fractional derivative exists.

Proof Based on Eq. (4), one can write

$$
\begin{aligned}
\left\|u_{n}(x, y, t)\right\| & \leq \sum_{i=0}^{n}\left\|e_{i}(x, y, t)\right\| \\
& \leq \sum_{i=0}^{n}\left(\frac{2(1-\alpha)}{(2-\alpha) M(\alpha)} \lambda+\frac{2 \alpha}{(2-\alpha) M(\alpha)} \lambda t\right)^{i}\|u(x, y, 0)\| .
\end{aligned}
$$


For $t=t_{0}$, one obtains

$$
\left\|u_{n}(x, y, t)\right\| \leq\|u(x, y, 0)\| \sum_{i=0}^{n}\left(\frac{2(1-\alpha)}{(2-\alpha) M(\alpha)} \lambda+\frac{2 \alpha}{(2-\alpha) M(\alpha)} \lambda t_{0}\right)^{i}
$$

Consequently,

$$
\lim _{n \rightarrow \infty}\left\|u_{n}(x, y, t)\right\| \leq\|u(x, y, 0)\| \sum_{i=0}^{\infty}\left(\frac{2(1-\alpha)}{(2-\alpha) M(\alpha)} \lambda+\frac{2 \alpha}{(2-\alpha) M(\alpha)} \lambda t_{0}\right)^{i}
$$

Since

$$
0 \leq \frac{2(1-\alpha)}{(2-\alpha) M(\alpha)} \lambda+\frac{2 \alpha}{(2-\alpha) M(\alpha)} \lambda t_{0}<1
$$

the above series is convergent, and therefore, $u_{n}(x, y, t)$ exists and is bounded for any $n$.

Besides, by assuming

$$
R_{n}(x, y, t)=u(x, y, t)-u_{n}(x, y, t)
$$

one can easily prove that

$$
\left\|R_{n}(x, y, t)\right\| \leq\left(\frac{2(1-\alpha)}{(2-\alpha) M(\alpha)} \lambda+\frac{2 \alpha}{(2-\alpha) M(\alpha)} \lambda t_{0}\right)^{n+1} \mu
$$

and so

$$
\lim _{n \rightarrow \infty}\left\|R_{n}(x, y, t)\right\| \leq \lim _{n \rightarrow \infty}\left(\frac{2(1-\alpha)}{(2-\alpha) M(\alpha)} \lambda+\frac{2 \alpha}{(2-\alpha) M(\alpha)} \lambda t_{0}\right)^{n+1} \mu
$$

But

$$
0 \leq \frac{2(1-\alpha)}{(2-\alpha) M(\alpha)} \lambda+\frac{2 \alpha}{(2-\alpha) M(\alpha)} \lambda t_{0}<1
$$

thus

$$
\lim _{n \rightarrow \infty}\left\|R_{n}(x, y, t)\right\|=0 \quad \text { or } \quad \lim _{n \rightarrow \infty} u_{n}(x, y, t)=u(x, y, t) .
$$

Theorem 4 If at $t=t_{0}$ we have

$$
0 \leq \frac{2(1-\alpha)}{(2-\alpha) M(\alpha)} \lambda+\frac{2 \alpha}{(2-\alpha) M(\alpha)} \lambda t_{0}<1,
$$

then the solution of the new $2 D-m K d V$ equation involving the CF time-fractional derivative is unique. 
Proof by contradiction To start, let us consider two solutions, say $u(x, y, t)$ and $v(x, y, t)$, for the model (1). One can write

$$
\begin{aligned}
u(x, y, t)-v(x, y, t)= & \frac{2(1-\alpha)}{(2-\alpha) M(\alpha)}(\psi(x, y, t ; u)-\psi(x, y, t ; v)) \\
& +\frac{2 \alpha}{(2-\alpha) M(\alpha)} \int_{0}^{t}(\psi(x, y, \varepsilon ; u)-\psi(x, y, \varepsilon ; v)) d \varepsilon .
\end{aligned}
$$

Consequently,

$$
\begin{aligned}
\|u(x, y, t)-v(x, y, t)\| \leq & \frac{2(1-\alpha)}{(2-\alpha) M(\alpha)}\|\psi(x, y, t ; u)-\psi(x, y, t ; v)\| \\
& +\frac{2 \alpha}{(2-\alpha) M(\alpha)} \int_{0}^{t}\|\psi(x, y, \varepsilon ; u)-\psi(x, y, \varepsilon ; v)\| d \varepsilon \\
\leq & \frac{2(1-\alpha)}{(2-\alpha) M(\alpha)} \lambda\|u-v\|+\frac{2 \alpha}{(2-\alpha) M(\alpha)} \int_{0}^{t} \lambda\|u-v\| d \varepsilon \\
= & \left(\frac{2(1-\alpha)}{(2-\alpha) M(\alpha)} \lambda+\frac{2 \alpha}{(2-\alpha) M(\alpha)} \lambda \int_{0}^{t} d \varepsilon\right)\|u-v\| \\
= & \left(\frac{2(1-\alpha)}{(2-\alpha) M(\alpha)} \lambda+\frac{2 \alpha}{(2-\alpha) M(\alpha)} \lambda t\right)\|u-v\| .
\end{aligned}
$$

But

$$
0 \leq \frac{2(1-\alpha)}{(2-\alpha) M(\alpha)} \lambda+\frac{2 \alpha}{(2-\alpha) M(\alpha)} \lambda t_{0}<1,
$$

therefore

$$
\|u(x, y, t)-v(x, y, t)\|=0,
$$

and so, the solution of the new $2 \mathrm{D}-\mathrm{mKdV}$ equation involving the CF time-fractional derivative is unique.

\section{The new CF time-fractional 2D-mKdV equation and its analytical solutions}

In the present section, first soliton solutions of the classical form of the model are extracted using an ansatz method, then the HAT method is used to acquire an analytic approximation for the new CF time-fractional 2D-mKdV equation.

\subsection{Soliton solutions of the classical form of the model}

To find soliton solutions, a test function is considered as follows:

$$
u(x, y, t)=A_{0}+A_{1} \operatorname{sech}\left(\alpha_{1} x+\alpha_{2} y+\alpha_{3} t\right) .
$$

By substituting the above function into the classical form of the model, we obtain a nonlinear algebraic system as follows:

$$
6 A_{0}^{2} \alpha_{1}-6 A_{0}^{2} \alpha_{2}+\alpha_{1}^{3}-3 \alpha_{1}^{2} \alpha_{2}+3 \alpha_{2}^{2} \alpha_{1}-\alpha_{2}^{3}-\alpha_{3}=0,
$$




$$
\begin{aligned}
& 12 A_{0} A_{1} \alpha_{1}-12 A_{0} A_{1} \alpha_{2}=0, \\
& 6 A_{1}^{2} \alpha_{1}-6 A_{1}^{2} \alpha_{2}-6 \alpha_{1}^{3}+18 \alpha_{1}^{2} \alpha_{2}-18 \alpha_{2}^{2} \alpha_{1}+6 \alpha_{2}^{3}=0,
\end{aligned}
$$

whose solution yields

$$
A_{0}=0, A_{1}=\mp \alpha_{1} \pm \alpha_{2}, \alpha_{3}=\alpha_{1}^{3}-3 \alpha_{1}^{2} \alpha_{2}+3 \alpha_{2}^{2} \alpha_{1}-\alpha_{2}^{3}
$$

Now, the following soliton solutions to the classical form of the model (1) can be constructed:

$$
u_{1,2}(x, y, t)=\left(\mp \alpha_{1} \pm \alpha_{2}\right) \operatorname{sech}\left(\alpha_{1} x+\alpha_{2} y+\left(\alpha_{1}^{3}-3 \alpha_{1}^{2} \alpha_{2}+3 \alpha_{2}^{2} \alpha_{1}-\alpha_{2}^{3}\right) t\right)
$$

For $\alpha_{1}=1$ and $\alpha_{2}=-1$, the above solitons are reduced to

$$
u_{1,2}(x, y, t)=\mp 2 \operatorname{sech}(x-y+8 t)=\mp 4 \frac{e^{x-y+8 t}}{1+e^{2(x-y+8 t)}}
$$

\subsection{The model and its analytic approximation}

To obtain an analytic approximation, we apply the Laplace transform to both sides of Eq. (1). Such an operation results in

$$
\begin{aligned}
\mathcal{L} & {[u(x, y, t)]-\frac{u(x, y, 0)}{s}-\left(\frac{s+\alpha(1-s)}{s}\right) \mathcal{L}\left[6 u^{2}(x, y, t) u_{x}(x, y, t)\right.} \\
& -6 u^{2}(x, y, t) u_{y}(x, y, t)+u_{x x x}(x, y, t)-u_{y y y}(x, y, t) \\
& \left.-3 u_{x x y}(x, y, t)+3 u_{x y y}(x, y, t)\right]=0 .
\end{aligned}
$$

Based on Eq. (5), the nonlinear operator can be defined as

$$
\begin{aligned}
\Omega[\phi(x, y, t ; p)]= & \mathcal{L}[\phi(x, y, t ; p)]-\frac{u_{0}(x, y, t)}{s} \\
& -\left(\frac{s+\alpha(1-s)}{s}\right) \mathcal{L}\left[6 \phi^{2}(x, y, t ; p) \phi_{x}(x, y, t ; p)\right. \\
& -6 \phi^{2}(x, y, t ; p) \phi_{y}(x, y, t ; p)+\phi_{x x x}(x, y, t ; p)-\phi_{y y y}(x, y, t ; p) \\
& \left.-3 \phi_{x x y}(x, y, t ; p)+3 \phi_{x y y}(x, y, t ; p)\right] \\
= & 0 .
\end{aligned}
$$

Now, the following $m$ th order deformation equation is considered:

$$
\mathcal{L}\left[u_{m}(x, y, t)-\chi_{m} u_{m-1}(x, y, t)\right]=h \mathcal{R}_{m}\left(\vec{u}_{m-1}\right)
$$

where

$$
\mathcal{R}_{m}\left(\vec{u}_{m-1}\right)=\left.\frac{1}{(m-1) !} \frac{\partial^{m-1} \Omega[\phi(x, y, t ; p)]}{\partial p^{m-1}}\right|_{p=0},
$$


and

$$
\chi_{m}= \begin{cases}0 & m \leq 1 \\ 1 & m>1\end{cases}
$$

It is worth mentioning that by selecting

$$
u(x, y, 0)=-4 \frac{e^{x-y}}{1+e^{2(x-y)}}
$$

and solving the resulting equations, one obtains

$$
\begin{aligned}
& u_{0}(x, y, t)=-4 \frac{e^{x-y}}{1+e^{2(x-y)}}, \\
& u_{1}(x, y, t)=-32 \frac{h e^{x-y}\left(\alpha e^{2(x-y)} t-\alpha e^{2(x-y)}+e^{2(x-y)}-\alpha t+\alpha-1\right)}{\left(e^{2(x-y)}+1\right)^{2}}
\end{aligned}
$$

Therefore, the series solution of the new CF time-fractional 2D-mKdV equation is derived as

$$
u(x, y, t)=-4 \frac{e^{x-y}}{1+e^{2(x-y)}}-32 \frac{h e^{x-y}\left(\alpha e^{2(x-y)} t-\alpha e^{2(x-y)}+e^{2(x-y)}-\alpha t+\alpha-1\right)}{\left(e^{2(x-y)}+1\right)^{2}}+\cdots
$$

It is noteworthy that for $\alpha=1$ and $h=-1$, the above series converges to the following exact solution:

$$
u(x, y, t)=-4 \frac{e^{x-y+8 t}}{1+e^{2(x-y+8 t)}}
$$

Figure 1 presents the 3-order approximation of the new CF time-fractional 2D-mKdV equation for $\alpha=1,0.99$, and 0.98 against the exact solution. From this figure, a full agreement between the 3-order approximation (when $\alpha=1$ ) and the exact solution is obviously observed. The absolute error of the 3-order approximation (when $\alpha=1$ ) and the exact solution has been presented in Table 1 . The results confirm the efficiency of the HAT method

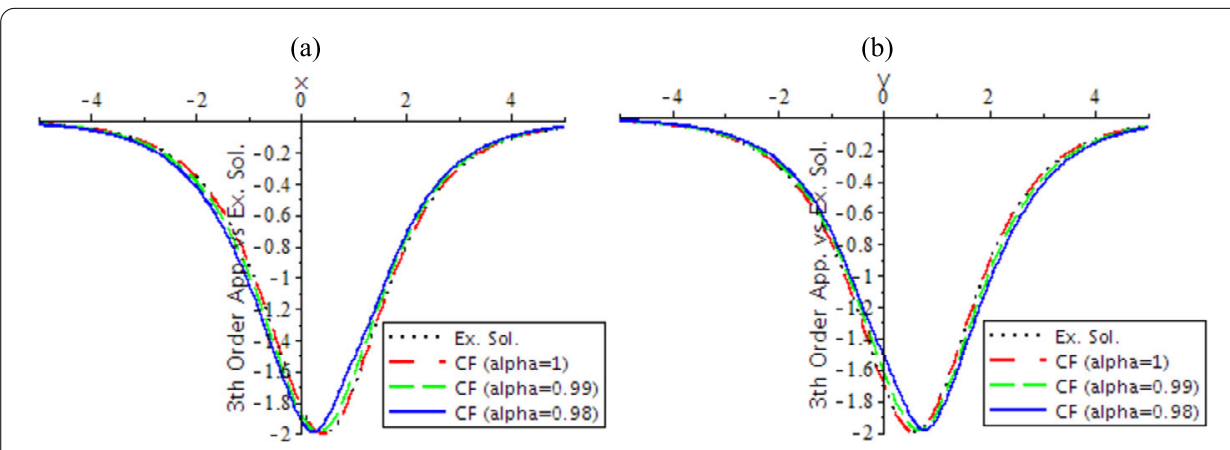

Figure 1 The 3th order approximation against the exact solution (a) $y=0.5, t=0.01, h=-1$, and $\alpha=1,0.99$, and 0.98 ; (b) $x=0.5, t=0.01, h=-1$, and $\alpha=1,0.99$, and 0.98 
Table 1 The absolute error of the 3-order approximation and the exact solution

\begin{tabular}{lll}
\hline$t$ & $\begin{array}{l}\text { Absolute error when } y=0.5, t=0.01, \\
h=-1, \text { and } \alpha=1\end{array}$ & $\begin{array}{l}\text { Absolute error when } x=0.5, t=0.01, \\
h=-1, \text { and } \alpha=1\end{array}$ \\
\hline 0 & $9.921881 \times 10^{-7}$ & $2.607245 \times 10^{-7}$ \\
0.5 & $1.702236 \times 10^{-5}$ & $1.702236 \times 10^{-5}$ \\
1 & $2.607245 \times 10^{-7}$ & $9.921881 \times 10^{-7}$ \\
1.5 & $6.913822 \times 10^{-6}$ & $7.076190 \times 10^{-6}$ \\
2 & $2.534162 \times 10^{-6}$ & $2.783063 \times 10^{-6}$ \\
2.5 & $2.305783 \times 10^{-7}$ & $3.045477 \times 10^{-7}$ \\
3 & $2.741436 \times 10^{-7}$ & $2.651408 \times 10^{-7}$ \\
3.5 & $2.706178 \times 10^{-7}$ & $2.751423 \times 10^{-7}$ \\
4 & $1.884674 \times 10^{-7}$ & $1.936185 \times 10^{-7}$ \\
4.5 & $1.198279 \times 10^{-7}$ & $1.235044 \times 10^{-7}$ \\
5 & $7.391757 \times 10^{-8}$ & $7.627198 \times 10^{-8}$ \\
\hline
\end{tabular}

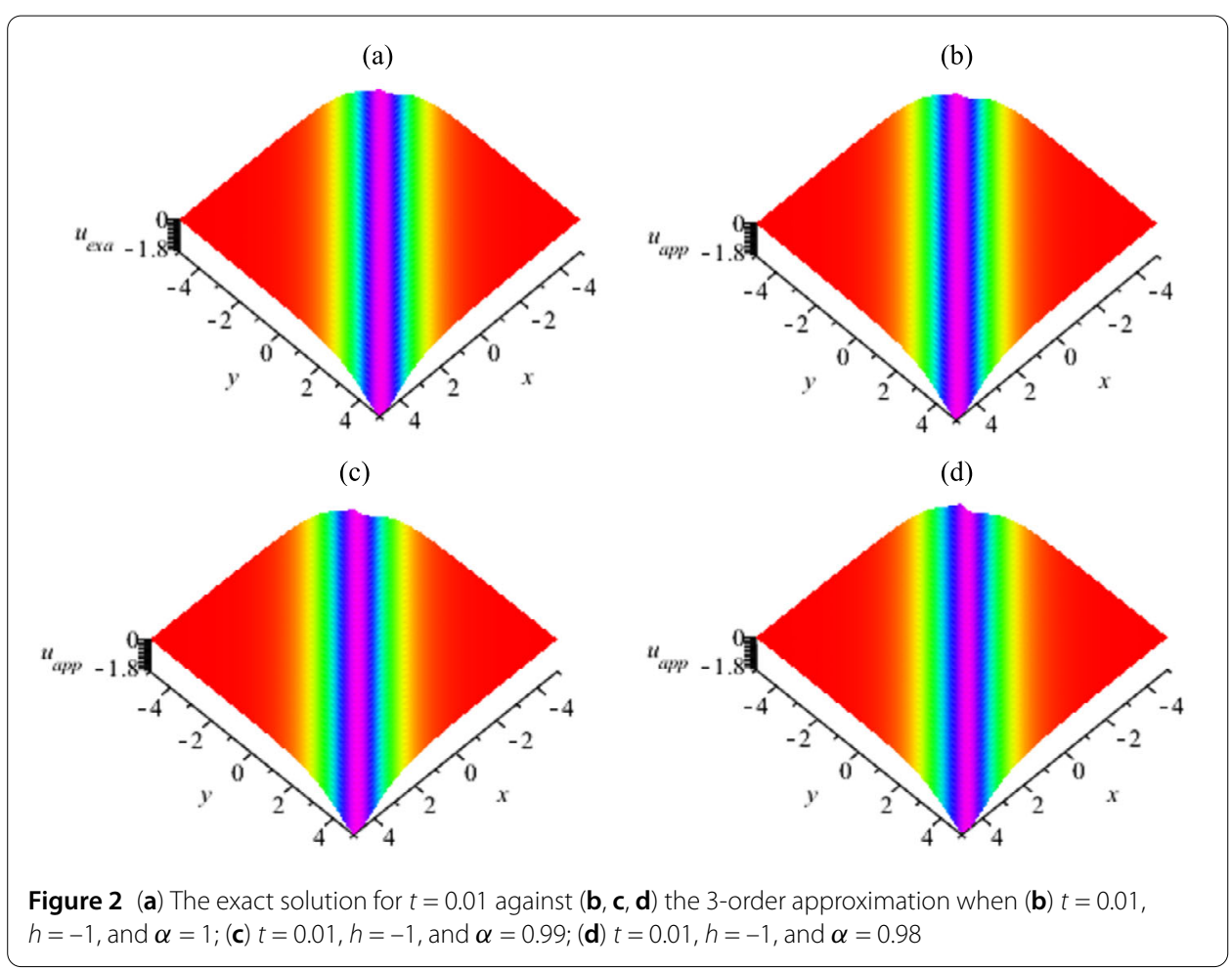

in deriving an analytic approximation with high accuracy. Finally, Fig. 2 shows the 3D plots of the exact solution and the 3-order approximation for $\alpha=1,0.99$, and 0.98 .

It is believed that the analytic approximation given by the HAT method can precisely predict the dynamics of the dark soliton solution of the new $2 \mathrm{D}-\mathrm{mKdV}$ equation involving the CF time-fractional derivative.

\section{Conclusion}

A thorough study on a nonlinear model involving the Caputo-Fabrizio time-fractional derivative was carried out successfully in the current paper. In this respect, a new 2D$\mathrm{mKdV}$ equation designed with the CF time-fractional derivative was considered, and an analytic approximation for it was formally derived using a systematic approach, named the homotopy analysis transform method. The existence and uniqueness of the solution of the new 2D-mKdV equation involving the Caputo-Fabrizio time-fractional derivative 
were studied by proving the Lipschitz condition for the kernel $\psi(x, y, t ; u)$ and applying the fixed-point theorem. A detailed study was finally accomplished to investigate the effect of the Caputo-Fabrizio operator on the dynamics of the obtained analytic approximation. The results presented herein confirm the efficiency of the HAT method in deriving an analytic approximation with high accuracy for nonlinear models involving the CaputoFabrizio time-fractional derivative. Our future work is to obtain an analytic approximation of the new 2D-mKdV equation with the Atangana-Baleanu time-fractional derivative and study the effect of the Atangana-Baleanu operator on the dynamics of the approximate solution.

\section{Acknowledgements \\ Not applicable.}

Funding

There is no funding for this work.

Availability of data and materials

Not applicable.

\section{Competing interests}

The authors declare that they have no competing interests.

Authors' contributions

All authors jointly worked on the results and they read and approved the final manuscript.

\section{Author details}

'Department of Mathematics, Rasht Branch, Islamic Azad University, Rasht, Iran. ${ }^{2}$ Department of Engineering Sciences, Faculty of Technology and Engineering, East of Guilan, University of Guilan, P.C. 44891-63157, Rudsar-Vajargah, Iran.

${ }^{3}$ Department of Mathematics, Faculty of Arts and Sciences, Cankaya University, Ankara 06530, Turkey. ${ }^{4}$ Institute of Space Sciences, Magurele-Bucharest, R 76900, Romania.

\section{Publisher's Note}

Springer Nature remains neutral with regard to jurisdictional claims in published maps and institutional affiliations.

Received: 2 April 2020 Accepted: 21 June 2020 Published online: 06 July 2020

\section{References}

1. https://en.wikipedia.org/wiki/Korteweg-de_Vries_equation

2. Wazwaz, A.M.: New sets of solitary wave solutions to the $\mathrm{KdV}, \mathrm{mKdV}$, and the generalized KdV equations. Commun. Nonlinear Sci. Numer. Simul. 13, 331-339 (2008)

3. Wang, C.: Spatiotemporal deformation of lump solution to $(2+1)$-dimensional KdV equation. Nonlinear Dyn. 84 , 697-702 (2016)

4. Sontakke, B.R., Shaikh, A.: The new iterative method for approximate solutions of time fractional $K d V, K(2,2)$, Burgers and cubic Boussinesq equations. Asian Res. J. Math. 1, 1-10 (2016)

5. Sontakke, B.R., Shaikh, A., Nisar, K.S.: Approximate solutions of a generalized Hirota-Satsuma coupled KdV and a coupled mKdV systems with time fractional derivatives. Malaysian J. Math. Sci. 12,175-196 (2018)

6. Saad, K.M., AL-Shareef, E.H.F., Alomari, A.K., Baleanu, D., Gómez-Aguilar, J.F.: On exact solutions for time-fractional Korteweg-de Vries and Korteweg-de Vries-Burger's equations using homotopy analysis transform method. Chin. J. Phys. 63, 149-162 (2020)

7. Bhatter, S., Mathur, A., Kumar, D., Nisar, K.S., Singh, J.: Fractional modified Kawahara equation with Mittag-Leffler law. Chaos Solitons Fractals 131, 109508 (2020)

8. Kumar, D., Singh, J., Kumar, S., Sushil: Numerical computation of Klein-Gordon equations arising in quantum field theory by using homotopy analysis transform method. Alex. Eng. J. 53, 469-474 (2014)

9. Yépez-Martínez, H., Gómez-Aguilar, J.F.: A new modified definition of Caputo-Fabrizio fractional-order derivative and their applications to the multi step homotopy analysis method (MHAM). J. Comput. Appl. Math. 346, 247-260 (2019)

10. Baleanu, D., Mohammadi, H., Rezapour, S.: Analysis of the model of HIV-1 infection of CD4 ${ }^{+}$T-cell with a new approach of fractional derivative. Adv. Differ. Equ. 2020, 71 (2020)

11. Baleanu, D., Jajarmi, A., Mohammadi, H., Rezapour, S.: A new study on the mathematical modelling of human liver with Caputo-Fabrizio fractional derivative. Chaos Solitons Fractals 134, 109705 (2020)

12. Wang, G., Kara, A.H.: A (2 + 1)-dimensional KdV equation and $\mathrm{mKdV}$ equation: symmetries, group invariant solutions and conservation laws. Phys. Lett. A 383, 728-731 (2019)

13. Shah, K., Jarad, F., Abdeljawad, T.: On a nonlinear fractional order model of Dengue fever disease under Caputo-Fabrizio derivative. Alex. Eng. J. (2020). https://doi.org/10.1016/j.aej.2020.02.022

14. Owolabi, K.M., Atangana, A.: Numerical approximation of nonlinear fractional parabolic differential equations with Caputo-Fabrizio derivative in Riemann-Liouville sense. Chaos Solitons Fractals 99, 171-179 (2017) 
15. Arshad, S., Defterli, O., Baleanu, D.: A second order accurate approximation for fractional derivatives with singular and non-singular kernel applied to a HIV model. Appl. Math. Comput. 374, 125061 (2020)

16. Shaikh, A., Tassaddiq, A., Nisar, K.S., Baleanu, D.: Analysis of differential equations involving Caputo-Fabrizio fractional operator and its applications to reaction-diffusion equations. Adv. Differ. Equ. 2019, 178 (2019)

17. Caputo, M., Fabrizio, M.: A new definition of fractional derivative without singular kernel. Prog. Fract. Differ. Appl. 1, 73-85 (2015)

18. Losada, J., Nieto, J.J.: Properties of a new fractional derivative without singular kernel. Prog. Fract. Differ. Appl. 1, 87-92 (2015)

19. Alizadeh, S., Baleanu, D., Rezapour, S.: Analyzing transient response of the parallel RCL circuit by using the Caputo-Fabrizio fractional derivative. Adv. Differ. Equ. 2020, 55 (2020)

20. Algahtani, O.J.J: Comparing the Atangana-Baleanu and Caputo-Fabrizio derivative with fractional order: Allen Cahn model. Chaos Solitons Fractals 89, 552-559 (2016)

21. Ali, F., Ali, F., Sheikh, N.A., Khan, I., Nisar, K.S.: Caputo-Fabrizio fractional derivatives modeling of transient MHD Brinkman nanoliquid: applications in food technology. Chaos Solitons Fractals 131, 109489 (2020)

22. Shaikh, A.S., Nisar, K.S.: Transmission dynamics of fractional order Typhoid fever model using Caputo-Fabrizio operator. Chaos Solitons Fractals 128, 355-365 (2019)

23. Atangana, A., Baleanu, D.: New fractional derivative with nonlocal and non-singular kernel, theory and application to heat transfer model. Therm. Sci. 20, 763-769 (2016)

24. Inc, M., Yusuf, A., Aliyu, A.I., Baleanu, D.: Investigation of the logarithmic-KdV equation involving Mittag-Leffler type kernel with Atangana-Baleanu derivative. Physica A 506, 520-531 (2018)

25. Korpinar, Z., Inc, M., Bayram, M.: Theory and application for the system of fractional Burger equations with Mittag-Leffler kernel. Appl. Math. Comput. 367, 124781 (2020)

26. Morales-Delgado, V.F., Gómez-Aguilar, J.F., Saad, K.M., Khan, M.A., Agarwal, P.: Analytic solution for oxygen diffusion from capillary to tissues involving external force effects: a fractional calculus approach. Physica A 523, 48-65 (2019)

27. Saad, K.M., Gómez-Aguilar, J.F.: Analysis of reaction-diffusion system via a new fractional derivative with non-singular kernel. Physica A 509, 703-716 (2018)

28. Veeresha, P., Prakasha, D.G.: Solution for fractional generalized Zakharov equations with Mittag-Leffler function. Results Eng. 5, 100085 (2020)

29. Gao, W., Veeresha, P., Prakasha, D.G., Baskonus, H.M., Yel, G.: A powerful approach for fractional Drinfeld-Sokolov-Wilson equation with Mittag-Leffler law. Alex. Eng. J. 58, 1301-1311 (2019)

30. Owolabi, K.M., Atangana, A.: Computational study of multi-species fractional reaction-diffusion system with ABC operator. Chaos Solitons Fractals 128, 280-289 (2019)

31. Heydari, M.H., Atangana, A., Avazzadeh, Z., Mahmoudi, M.R.: An operational matrix method for nonlinear variable-order time fractional reaction-diffusion equation involving Mittag-Leffler kernel. Eur. Phys. J. Plus 135, 237 (2020)

32. Jajarmi, A., Baleanu, D.: A new fractional analysis on the interaction of HIV with CD4 + T-cells. Chaos Solitons Fractals $113,221-229(2018)$

33. Yusuf, A., Inc, M., Aliyu, A.I., Baleanu, D.: Efficiency of the new fractional derivative with nonsingular Mittag-Leffler kernel to some nonlinear partial differential equations. Chaos Solitons Fractals 116, 220-226 (2018)

34. Liao, S.: Beyond Perturbation: Introduction to the Homotopy Analysis Method. CRC Press, Boca Raton (2004)

35. Samadani, F., Moradweysi, P., Ansari, R., Hosseini, K., Darvizeh, A.: Application of homotopy analysis method for the pull-in and nonlinear vibration analysis of nanobeams using a nonlocal Euler-Bernoulli beam model. Z. Naturforsch. A 72, 1093-1104 (2017)

36. Zabihi, A., Ansari, R., Hosseini, K., Samadani, F., Torabi, J.: Nonlinear pull-in instability of rectangular nanoplates based on the positive and negative second-order strain gradient theories with various edge supports. Z. Naturforsch. A, J. Phys. Sci. 75, 317-331 (2020)

37. Torabi, J., Ansari, R., Zabihi, A., Hosseini, K.: Dynamic and pull-in instability analyses of functionally graded nanoplates via nonlocal strain gradient theory. Mech. Based Des. Struct. Mach. (2020). https://doi.org/10.1080/15397734.2020.1721298

38. Hosseini, S.M.J., Ansari, R., Torabi, J., Zabihi, A., Hosseini, K.: Nonlocal strain gradient pull-in study of nanobeams considering various boundary conditions. Iran. J. Sci. Technol., Trans. Mech. Eng. (2020). https://doi.org/10.1007/s40997-020-00365-6

39. Sontakke, B.R., Shaikh, A.: Approximate solutions of time fractional Kawahara and modified Kawahara equations by fractional complex transform. Commun. Numer. Anal. 2016, 218-229 (2016)

40. Sontakke, B.R., Shaikh, A., Nisar, K.S.: Existence and uniqueness of integrable solutions of fractional order initial value equations. J. Math. Model. 6, 137-148 (2018)

41. Nisar, K.S.: Generalized Mittag-Leffler type function: fractional integrations and application to fractional kinetic equations. Front. Phys. 8, 33 (2020)

42. Khan, O., Khan, N., Baleanu, D., Nisar, K.S.: Computable solution of fractional kinetic equations using Mathieu-type series. Adv. Differ. Equ. 2019, 234 (2019)

43. Khan, O., Khan, N., Nisar, K.S., Saif, M., Baleanu, D.: Fractional calculus of a product of multivariable Srivastava polynomial and multi-index Bessel function in the kernel $F_{3}$. AIMS Math. 5, 1462-1475 (2020)

44. Jumani, T.A., Mustafa, M.W., Hussain, Z., Rasid, M.M., Saeed, M.S., Memon, M.M., Khan, I., Nisar, K.S.: Jaya optimization algorithm for transient response and stability enhancement of a fractional-order PID based automatic voltage regulator system. Alex. Eng. J. (2020). https://doi.org/10.1016/j.aej.2020.03.005

45. Yusuf, A., Inc, M., Aliyu, A.l., Baleanu, D.: Conservation laws, soliton-like and stability analysis for the time fractional dispersive long-wave equation. Adv. Differ. Equ. 2018, 319 (2018) 